\title{
LONG-TERM RESULTS OF THE LATERAL TUNNEL FONTAN OPERATION
}

Christof Stamm, MD

Ingeborg Friehs, MD

John E. Mayer, Jr, MD

David Zurakowski, $\mathrm{PhD}^{\mathrm{b}}$

John K. Triedman, MD

Adrian M. Moran, MD $^{\mathrm{a}}$

Edward P. Walsh, MD

James E. Lock, MD

Richard A. Jonas, MD

Pedro J. del Nido, MD
Objectives: Completion of a total cavopulmonary anastomosis with an intraatrial lateral tunnel is known to yield good early and midterm results. In this study, we sought to determine the long-term outcome (10 years) after a lateral tunnel Fontan procedure.

Methods: Between October 1987 and December 1991, 220 patients (aged 11 months to 32 years) with a wide range of underlying diagnoses underwent a fenestrated or nonfenestrated lateral tunnel Fontan procedure at our institution. Current follow-up information was available for 196 patients (94\%, mean follow-up $=10.2 \pm 0.6$ years $)$. Risk factor analysis included patientrelated and procedure-related variables, with death, failure, and bradyarrhythmia or tachyarrhythmia as outcome parameters.

Results: There were 12 early deaths ( $<30$ days or hospital death), 7 late deaths, 4 successful takedown operations, and 4 heart transplantations. Kaplan-Meier estimated survival was $93 \%$ at 5 years and $91 \%$ at 10 years, and freedom from failure was $90 \%$ at 5 years and $87 \%$ at 10 years. Freedom from new supraventricular tachyarrhythmia was $96 \%$ at 5 years and $91 \%$ at 10 years; freedom from new bradyarrhythmia was $88 \%$ at 5 years and $79 \%$ at 10 years. Three patients had evidence of protein-losing enteropathy. Multivariable risk factors for development of supraventricular tachyarrhythmia included heterotaxy syndrome, atrioventricular valve abnormalities, and preoperative bradyarrhythmia. Risk factors for bradyarrhythmia included systemic venous anomalies. The sole risk factor for late failure was a previous coarctation repair.

Conclusion: The lateral tunnel Fontan procedure results in excellent longterm outcome even when used in patients with diverse anatomic diagnoses. The incidence of atrial tachyarrhythmia is low and mainly depends on the underlying cardiac morphology and preoperative arrhythmia. The good longterm outcome after an intracardiac lateral tunnel Fontan procedure should serve as a basis for comparison with other surgical alternatives. ( $\mathrm{J}$ Thorac Cardiovasc Surg 2001;121:28-41)
From the Children's Hospital Boston, Harvard Medical School, Departments of Cardiac Surgery, Cardiology, and Biostatistics, ${ }^{b}$ Boston, Mass.

Part of this work was done while Dr Moran was the Tommy Kaplan Fellow in the Children's Hospital Cardiovascular Program.

Read at the Eightieth Annual Meeting of The American Association for Thoracic Surgery, Toronto, Ontario, Canada, April 30-May 3, 2000.

Received for publication May 24, 2000; revisions requested July 13, 2000; revisions received Aug 2, 2000; accepted for publication Sept 8, 2000.

Address for reprints: Pedro J. del Nido, MD, Children's Hospital Boston, Cardiac Surgery, 300 Longwood Ave, Boston, MA 02115 (E-mail: delnido@a1.tch.harvard.edu).

Copyright (C) 2001 by The American Association for Thoracic Surgery

$0022-5223 / 2001 \$ 35.00+0 \quad \mathbf{1 2 / 6 / 1 1 1 4 2 2}$

doi: $10.1067 / \mathrm{mtc} .2001 .111422$
Qince Fontan and Baudet's report ${ }^{1}$ of the first successful right heart bypass directing the entire systemic venous blood flow to the pulmonary arteries in a patient with tricuspid atresia, a large amount of experimental and surgical work has been done to improve the prognosis of patients in whom a biventricular repair cannot be achieved. Once the feasibility of pulmonary arterial perfusion without a pumping chamber was demonstrated clinically, several techniques to connect the systemic venous atrium with the central pulmonary arteries were developed. ${ }^{2}$ However, intermediate and long-term results soon showed that incorporation of the entire systemic venous atrium in the Fontan circulation was associated with a high incidence of supraventricular tachyarrhythmias (SVTs) and thromboembolic complications. ${ }^{3-7}$ 
Turbulent blood flow in the systemic venous atrium with parietal stasis increases the risk of thrombus formation, and elevated atrial pressure facilitates distention of the atrial wall, progressive right atrial enlargement, and conduction abnormalities. Hence, de Leval and associates ${ }^{8}$ and Jonas and Castaneda ${ }^{9}$ developed a technique to guide the systemic venous return from the inferior vena cava (IVC) to the orifice of the superior vena cava (SVC) through an intra-atrial tunnel that consists of a prosthetic baffle and incorporates a portion of the lateral atrial wall. The concept was to achieve laminar blood flow in a tubular construct and to provide growth potential, as well as a source for endothelialization of the synthetic surface. The lateral tunnel Fontan operation has since been performed in large series with low operative and intermediate mortality, but little information is available regarding the long-term outcome. To date, studies of long-term results after Fontan operations are mainly based on patients with direct atriopulmonary anastomoses and rarely allow a valid assessment of the long-term outcome after a lateral tunnel Fontan procedure. ${ }^{4,6,10}$ A study covering the first 500 patients undergoing any modification of the Fontan operation at our institution included our initial experience with a lateral tunnel Fontan operation, but only short-term follow-up was available for this subgroup of patients. ${ }^{11,12}$ In view of the current controversy regarding the indications for a total extracardiac Fontan procedure, we now sought to determine the long-term outcome of the first 220 patients who underwent a lateral tunnel Fontan procedure at Children's Hospital Boston.

\section{Methods}

Patients undergoing a total cavopulmonary anastomosis with an intra-atrial lateral tunnel at Children's Hospital Boston between October 1987 and December 1991 were identified from the databases of the Departments of Cardiac Surgery and Cardiology. This cohort includes patients with an interrupted IVC and azygos continuation in whom the hepatic venous blood flow was directed to the orifice of the SVC by means of a typical lateral intra-atrial tunnel. Four patients who underwent a lateral tunnel Fontan operation as a conversion from a previous atriopulmonary anastomosis were excluded. ${ }^{13}$ Also excluded were 3 patients in whom an intraatrial lateral tunnel guides blood flow from the IVC to the entrance of a right-sided atrial appendage, which was connected with the pulmonary arteries distally.

Medical records, operative notes, all available electrocardiograms (ECGs), echocardiography reports, and cardiac catheterization reports were reviewed. In cases in which no current follow-up information was available, primary physicians and cardiologists monitoring the patient were contacted. Preoperative data were partly incorporated in previous studies from our institution, but all data were re-evaluated because the group termed total cavopulmonary anastomosis in the studies by Gentles, ${ }^{10}$ Fishberger, ${ }^{12}$ and their associates also included a number of patients in whom no lateral tunnel was inserted but instead the atrial septal defect was closed or the pulmonary venous return routed to the atrioventricular valve(s) by means of a complex intra-atrial patch.

Surgical technique. After median sternotomy, bicaval cannulation, and aortic cannulation, all patients were placed on hypothermic cardiopulmonary bypass. Aortic crossclamping and cardioplegic arrest were performed in all but 5 patients. The pulmonary artery was divided, the proximal end closed, any systemic-pulmonary arterial shunts taken down, and a pulmonary arterioplasty was performed when indicated. The rightsided atrium was then opened parallel to the sulcus terminalis but more anterior than for other intra-atrial procedures, and the atrial septum was excised within the confines of the limbus of the fossa ovalis to ensure unobstructed communication between the atria. A polytetrafluoroethylene (PTFE) ${ }^{*}$ cardiovascular patch or a PTFE tube graft (10-18 mm diameter) that had been opened longitudinally was inserted so as to direct blood flow from the IVC to the orifice of the SVC or superior end of the right atrium, incorporating a portion of the lateral atrial wall in the newly constructed tunnel. The exact position of the suture line was not always clear from the operative report, and some variations may have occurred as a result of anatomic features and the surgeon's preference. In general, the suture starts between the orifices of the IVC and coronary sinus and is carried toward the SVC orifice, staying anterior to the posterior rim of the atrial septum to avoid pulmonary venous obstruction. Around the SVC orifice, sutures are placed into the nontrabeculated tissue at the SVC entrance site, posterior to the crista terminalis. In cases in which the crista terminalis has to be used for secure anchorage of the patch, stitches are placed superficially to prevent damage to conduction pathways following the crista terminalis muscle bundles. The suture is then carried along the lateral atrial wall toward the IVC orifice, then around the IVC orifice, leaving the coronary sinus on the pulmonary venous atrial side of the baffle. In a subgroup of patients a small (3-4 $\mathrm{mm}$ ) fenestration was created in the PTFE baffle with a coronary punch. Finally, the cavopulmonary anastomosis was constructed, the technique depending on the anatomy of the SVC and the presence of a prior bidirectional Glenn shunt.

Definitions. Supraventricular tachyarrhythmia (SVT) was defined as the presence of documented atrial fibrillation, atrial flutter, or atrioventricular reciprocating tachycardia, but not sinus tachycardia. The definition of bradyarrhythmia included sinus bradycardia, abrupt and pronounced sinus pause or arrest, sick sinus syndrome, ${ }^{14}$ slow junctional escape rhythm, junctional rhythm at physiologic rates without evidence of sinus node activity, and second- or third-degree atrioventricular block. Sinus bradycardia was defined as a resting heart rate of more than 2 standard deviations less than the age-adjusted mean. For patients followed up at our institution, the presence of arrhythmia was confirmed by reviewing the ECG recording. For patients who underwent follow-up examinations elsewhere, all arrhythmias that were reported in the correspondence were noted, without an attempt to deter-

*Gore-Tex patch or graft; registered trade mark of W. L Gore \& Associates, Inc, Flagstaff, Ariz. 
mine significance. New postoperative arrhythmia was defined as the occurrence of arrhythmia after the Fontan operation, including the early postoperative period, in patients without evidence of preoperative arrhythmia. Lateonset arrhythmia was defined as the occurrence of arrhythmia during the follow-up period in patients without evidence of preoperative or early postoperative arrhythmia. The early postoperative period was defined as the time before discharge from the hospital or the first 30 postoperative days for patients who were hospitalized for more than 30 postoperative days.

Statistical analysis. Variables tested for influence on the outcome parameters are listed in the appendix. Outcome parameters were overall death, early death, late death, overall Fontan failure (defined as death, takedown, or transplantation), early failure, late failure, presence of early and late SVT, and presence of early and late bradyarrhythmia. The incidences of new postoperative bradyarrhythmia or SVT were analyzed as separate outcomes. Estimated actuarial survival and freedom from failure, SVT, and bradyarrhythmia were determined by the Kaplan-Meier product-limit method with $95 \%$ confidence intervals (CIs) calculated by means of the Greenwood formula. ${ }^{15}$ Significance was assessed in the univariable analysis by the log-rank test for late and total outcomes and by the likelihood ratio $\chi^{2}$ test for early outcomes. For multivariable analysis, the Cox proportional-hazards regression model was used to establish the variables independently predictive of late and total survival, Fontan failure, and arrhythmias. For outcomes occurring during the early postoperative period, stepwise logistic regression analysis was performed. Hazard ratios and odds ratios with $95 \%$ confidence intervals were constructed for the significant multivariable predictors. Final models were derived by the forward stepwise selection procedure with variables having $P<.1$ in the univariable analyses entered as candidates into the Cox and multiple logistic regression models; a $P$ value $<.05$ was required for a variable to be retained in the equation. To account for the often transient nature of SVT, we compared the proportion of patients having SVT at most recent followup with those having preoperative SVT by the Fisher exact test. All reported $P$ values are 2-tailed. Statistical analysis was performed by means of the SPSS software package (version 9.0, SPSS, Inc, Chicago, Ill).

\section{Results}

Patient characteristics. A total of 220 consecutive patients were included in the study. The median age at the time of the operation was 3.9 years (range 11 months to 27 years). A total of 128 patients were male and 92 were female. The major anatomic diagnoses included tricuspid atresia in 32 patients and doubleinlet left ventricle in 52 patients. To facilitate data analysis based on the morphology of the predominant ventricle and its relation to the great arteries, we assigned the diagnoses to one of the following groups: single left ventricle with normally related great arteries $(n=63)$, single left ventricle with transposed great arteries $(\mathrm{n}=81)$, single right ventricle excluding hypoplastic left heart syndrome $(n=26)$, hypoplastic left heart syndrome $(n=15)$, heterotaxy syndrome $(n=22)$, and others $(n=13)$. Mild or moderate atrioventricular valve regurgitation was present in 38 (14\%) patients. A total of 47 patients had anomalies of systemic $(n=40)$ or pulmonary $(n=12)$ venous return.

One hundred eighty-seven patients had previously undergone a total of 348 palliative procedures: 130 patients had a previous systemic-pulmonary artery shunt, 38 patients had a bidirectional and 13 a classic Glenn shunt, and 37 of those had a systemic-pulmonary artery shunt followed by a bidirectional Glenn shunt. Thirty-two patients had a pulmonary artery-aortic anastomosis in the form of a Damus-Kaye-Stansel anastomosis $(\mathrm{n}=17)$ or Norwood stage I operation $(\mathrm{n}=15)$. Other previous procedures included atrial septectomy $(\mathrm{n}=41)$, pulmonary artery banding $(\mathrm{n}=51)$, pulmonary arterioplasty $(n=24)$, coarctation repair $(\mathrm{n}=12)$, and enlargement of a restrictive ventricular septal defect or bulboventricular foramen $(n=7)$.

Preoperative cardiac catheterization data were available in 198 patients and, in conjunction with echocardiographic data, served as the basis for assessment of atrial and ventricular morphology and hemodynamic data. Mean pulmonary artery pressure was $13.1 \pm 4.6$ $\mathrm{mm} \mathrm{Hg}$ (range 5-38 $\mathrm{mm} \mathrm{Hg}$ ), and mean indexed pulmonary arterial resistance was $1.7 \pm 0.9$ Wood units (range 0.4-6.8 Wood units). Four patients had an indexed pulmonary arterial resistance of more than 4 Wood units, and 9 patients had a pulmonary artery pressure of more than $20 \mathrm{~mm} \mathrm{Hg}$, but a significant response to high inspired oxygen was noted in most. Mean right atrial pressure was $6.5 \pm 3.2 \mathrm{~mm} \mathrm{Hg}$ (range 0-21 $\mathrm{mm} \mathrm{Hg}$ ), and mean ventricular end-diastolic pressure was $8.6 \pm 3.4 \mathrm{~mm} \mathrm{Hg}$ (range $0-26 \mathrm{~mm} \mathrm{Hg}$ ). Patients with elevated ventricular end-diastolic pressure commonly had increased ventricular volume load with preserved ventricular function. Mean arterial oxygen saturation was $81.5 \% \pm 6.6 \%$ (range 57\%-94\%), mean hematocrit value was $53.1 \% \pm 4.7 \%$ (range $41 \%$ $72 \%$ ), with a mean hemoglobin value of $16.7 \pm 2.1$ $\mathrm{mg} / \mathrm{dL}$ (range 11.8-26.3 mg/dL).

A total of 218 patients had preoperative ECGs, and 47 had undergone prior Holter recordings. In 2 patients the preoperative rhythm could not be determined on the basis of the available data. Preoperatively, 182 (83\%) patients were in normal sinus rhythm, and 27 patients were in bradyarrhythmia. Eleven patients had junctional rhythm at a physiologic heart rate, 8 of whom had undergone construction of a bidirectional Glenn shunt. Seven $(3 \%)$ patients had documented preoperative 
Table I. Early and late failures after the lateral tunnel Fontan operation

\begin{tabular}{|c|c|c|c|c|}
\hline Diagnosis & $\begin{array}{c}\text { Age at } \\
\text { operation (y) }\end{array}$ & Cause of failure & Interval (mo) & Outcome \\
\hline \multicolumn{5}{|c|}{ Early failure } \\
\hline DILV & 2.5 & Progressive pulmonary hypertension & 0.01 & Takedown \\
\hline HLHS & 2.8 & Low cardiac output & 0.01 & Death \\
\hline Single LV & 4.0 & Low cardiac output & 0.01 & Death \\
\hline TA, LPA occlusion & 1.7 & Cardiac arrest & 0.01 & Takedown \\
\hline DORV, hypoplastic LV & 3.4 & Ventricular dysfunction & 0.1 & Death \\
\hline HLHS & 2.1 & Cardiac arrest & 0.2 & Death \\
\hline HLHS & 0.9 & Low cardiac output/multiorgan failure & 0.2 & Takedown/death \\
\hline Single LV, TGA & 1.9 & Low cardiac output & 0.3 & Takedown/death \\
\hline DORV, hypoplastic LV & 2.6 & Low cardiac output/brain death & 0.5 & Takedown/death \\
\hline Single ventricle, D-TGA & 1.1 & Cerebral bleeding & 0.6 & Death \\
\hline Heterotaxy, single LV & 2.1 & SVC thrombosis, sepsis & 1.1 & Death \\
\hline Single RV & 6.9 & Ventricular dysfunction & 1.1 & Death \\
\hline Single LV, L-TGA & 4.1 & AVVR, sepsis & 1.2 & Death \\
\hline $\mathrm{TA}$ & 2.3 & Ventricular dysfunction & 2.7 & Death \\
\hline \multicolumn{5}{|c|}{ Late failure } \\
\hline TA, TGA & 2.5 & Persisting effusions & 3.0 & Takedown \\
\hline HLHS & 2.5 & SVC, PA thrombus & 6.3 & Death \\
\hline Single LV & 11.6 & Unknown & 8.2 & Death \\
\hline Heterotaxy, single LV & 7.4 & Baffle thrombosis & 8.9 & HTX \\
\hline TA, TGA & 2.8 & Cerebral thromboembolism & 21.4 & Death \\
\hline DORV, hypoplastic LV & 2.6 & Persisting effusions & 31.2 & Takedown \\
\hline DORV, hypoplastic LV & 3.9 & Ventricular arrhythmia & 32.3 & Death \\
\hline TGA, Ebstein's anomaly & 12.3 & Ventricular dysfunction, AVVR & 44.7 & HTX \\
\hline DORV, ccTGA & 21.3 & Ventricular dysfunction & 57.9 & HTX \\
\hline DORV, mitral atresia & 12.4 & Ventricular dysfunction, PLE & 63.8 & Death \\
\hline CAVC, hypoplastic LV & 9.2 & Ventricular dysfunction, AVVR & 89.4 & HTX \\
\hline Taussig-Bing complex & 3.1 & Ventricular dysfunction & 98.1 & Death \\
\hline DILV & 10.7 & Aortic regurgitation, ventricular dysfunction & 101.9 & Death \\
\hline
\end{tabular}

$D I L V$, Double-inlet left ventricle; $H L H S$, hypoplastic left heart syndrome; $L V$, left ventricle; $T A$, tricuspid atresia; $L P A$, left pulmonary artery; $D O R V$, double-outlet right ventricle; $T G A$, transposition of the great arteries; $R V$, right ventricle; $c c T G A$, congenitally corrected transposition of the great arteries; $C A V C$, complete atrioventricular connection; $S V C$, superior vena cava; $A V V R$, atrioventricular valve regurgitation; $P A$, pulmonary artery; $P L E$, protein-losing enteropathy; $H T X$, heart transplantation.

SVT, and $11(5 \%)$ patients had had a pacemaker implanted.

Surgical procedures. Three technical modifications of the lateral tunnel cavopulmonary anastomosis were used in this series. The SVC was divided and anastomosed with corresponding incisions in the right pulmonary artery in 116 patients. The right pulmonary artery was transected and anastomosed with longitudinal incisions in the SVC in 43 patients. In 62 patients the superior cavoatrial junction was incised after transection of the SVC. The roof of the right-sided atrium was then opened and the undersurface of the right pulmonary artery anastomosed to the orifice composed of the proximal SVC stump and the right atrial roof. This modification was developed to minimize the risk of development of a stenosis at the proximal SVC-pulmonary artery anastomosis in patients with an SVC orifice that was markedly smaller in diameter than the IVC. Fenestration of the intra-atrial baffle was performed in $140(63 \%)$ patients. Fenestration of the later- al tunnel graft was first performed in our institution in May 1989, so that all patients operated on before that date had a nonfenestrated intra-atrial baffle. In patients operated on after May 1989, the indication for baffle fenestration was mainly based on the presence of preoperative elevated pulmonary artery pressure and/or a pulmonary vascular resistance of more than 2 Wood units. Concomitant surgical procedures included pulmonary arterioplasty in 51 patients, atrial septectomy $(\mathrm{n}=144)$, and takedown of any systemic-pulmonary artery shunt. Additional intracardiac procedures included enlargement of a restrictive bulboventricular foramen in 7 patients and atrioventricular valve reconstruction in 2. Ablation of ectopic atrial foci was performed in 3 patients. The mean duration of cardiopulmonary bypass was $120 \pm 27$ minutes (range 70-273 minutes), and mean aortic crossclamping time was $59 \pm 20$ minutes (0-111 minutes). Cardiac arrest and myocardial protection were achieved with oxygenated glucose plus potassium cardioplegic solution $(n=62)$, St Thomas' 
Table II. Predictors of failure after lateral tunnel Fontan operation

\begin{tabular}{|c|c|c|c|c|c|}
\hline \multirow[b]{2}{*}{ Variable } & \multicolumn{3}{|c|}{ Univariable } & \multicolumn{2}{|c|}{ Multivariable } \\
\hline & Association & $\mathrm{P}$ value & Risk ratio* & $95 \% C I$ & $\mathrm{P}$ value \\
\hline \multicolumn{6}{|c|}{ Early failure } \\
\hline Age at Fontan operation & + & .005 & & & .18 \\
\hline LV-NRGA & + & .05 & & & .27 \\
\hline Single right ventricle & + & .08 & 5.1 & $1.2-20.8$ & .04 \\
\hline Hypoplastic left heart syndrome & + & .06 & & & .13 \\
\hline Right AVV stenosis or atresia & + & .07 & & & .29 \\
\hline Left AVV stenosis or atresia & + & .03 & & & .70 \\
\hline Pulmonary artery distortion & + & .01 & 11.7 & $2.8-49.3$ & .004 \\
\hline Prior atrial septectomy & + & .03 & & & .21 \\
\hline Prior coarctation repair & + & .08 & & & .39 \\
\hline Preop mean pulmonary artery pressure & + & .09 & & & .36 \\
\hline Preop pulmonary vascular resistance & + & .08 & 1.8 & $1.1-2.8$ & .04 \\
\hline Postop right atrial pressure & + & .003 & 1.4 & $1.1-1.9$ & .02 \\
\hline \multicolumn{6}{|c|}{ Late failure } \\
\hline AVV regurgitation & + & .03 & & & .55 \\
\hline Prior systemic-pulmonary shunt & - & $<.001$ & 0.07 & $0.02-0.45$ & .008 \\
\hline Prior pulmonary artery banding & + & .04 & & & .25 \\
\hline Prior coarctation repair & + & .05 & 13.5 & $5.1-35.9$ & $<.001$ \\
\hline Postop right atrial pressure & + & .002 & & & .62 \\
\hline Early postop bradyarrhythmia & + & .006 & & & .54 \\
\hline \multicolumn{6}{|c|}{ Total failure } \\
\hline Prior pulmonary artery banding & + & .04 & & & .25 \\
\hline Prior coarctation repair & + & .01 & & & .1 \\
\hline Prior bradyarrhythmia & + & .02 & & & .06 \\
\hline Postop right atrial pressure & + & .006 & 1.3 & $1.1-1.5$ & .005 \\
\hline Hypoplastic left heart syndrome & + & .07 & & & .86 \\
\hline
\end{tabular}

$C I$, Confidence interval; $L V-N R G A$, left ventricle with normally related great arteries; $A V V$, atrioventricular valve.

*Risk ratio represents the odds ratio derived from logistic regression analysis for the outcome "early failure" and hazards ratio from the Cox proportional-hazards model for the outcomes "late failure" and "total failure." Only variables with a univariate $P<.1$ are shown. All variables tested are listed in the appendix.

Hospital cardioplegic solution $(\mathrm{n}=87)$, or dilute blood, glucose, and potassium cardioplegic solution $(\mathrm{n}=67)$.

Early results. There were $12(5.4 \%)$ early deaths. The preoperative diagnoses and causes of death are shown in Table I. The most common cause for early death was ventricular dysfunction $(n=9)$. Two patients could not be weaned from bypass and died in circulatory failure. One patient died of an early postoperative cerebral hemorrhage, and 1 patient underwent early tricuspid replacement for severe regurgitation and died later of septic complications. None of the abovementioned patients had any structural anomaly of the Fontan pathway as demonstrated by postoperative catheterization, surgical exploration, or autopsy. However, 1 patient with heterotaxy syndrome and complex venous anatomy developed a thrombus in the SVC. The thrombus was surgically removed and the SVC enlarged with a patch, but he died subsequently of septic complications. In 2 patients the Fontan circulation was successfully taken down during the early postoperative period. One patient with tricuspid atresia, left pulmonary artery occlusion, but a well-developed right pulmonary artery had a cardiac arrest 2 hours postoperatively. He was supported with an extracorporeal membrane oxygenator, and the Fontan was later converted to a classic Glenn shunt and an aorta-left pulmonary artery shunt. Another patient had an increase in pulmonary vascular resistance with low cardiac output several hours after a fenestrated Fontan procedure, requiring conversion to a bidirectional Glenn shunt on the same day. Multivariable analysis by logistic regression identified single right ventricle, preoperative pulmonary artery distortion, higher preoperative pulmonary vascular resistance, and higher postoperative right atrial pressure as risk factors for early failure (Table II). When early death was considered as the outcome parameter, hypoplastic left heart syndrome (odds ratio $=4.8,95 \% \mathrm{CI}=1.4-16.8, P=.04$ ) and pulmonary artery distortion (odds ratio $=4.3,95 \% \mathrm{CI}=$ $1.5-12.5, P=.03$ ) proved the only independent risk factors.

Mean central venous pressure on the first postoperative day was $13.9 \pm 2.5 \mathrm{~mm} \mathrm{Hg}$ (range $7-25 \mathrm{~mm} \mathrm{Hg}$ ), and mean left atrial pressure was $6.8 \pm 2.2 \mathrm{~mm} \mathrm{Hg}$ (range 1-14 mm Hg). The median postoperative venti- 
Table III. Predictors of SVT after the lateral tunnel Fontan operation

\begin{tabular}{|c|c|c|c|c|c|}
\hline \multirow[b]{2}{*}{ Variable } & \multicolumn{3}{|c|}{ Univariable } & \multicolumn{2}{|c|}{ Multivariable } \\
\hline & Association & P value & Risk ratio* & $95 \% C I$ & $\mathrm{P}$ value \\
\hline \multicolumn{6}{|c|}{ Early SVT } \\
\hline Heterotaxy syndrome & + & .002 & 7.8 & $2.3-23.4$ & .01 \\
\hline Prior pulmonary artery banding & + & .08 & 10.0 & $1.5-66.2$ & .02 \\
\hline Preop SVT & + & $<.0001$ & 71.5 & $13.7-37.2$ & $<.0001$ \\
\hline \multicolumn{6}{|c|}{ Late SVT } \\
\hline Heterotaxy syndrome & + & .08 & & & .54 \\
\hline AVV regurgitation & + & .1 & 8.9 & $1.7-46.0$ & .03 \\
\hline Left AVV stenosis or atresia & + & .07 & & & .45 \\
\hline CAVV & + & .009 & 16.9 & 3.1-91.4 & .006 \\
\hline Anomalous systemic venous return & + & .09 & & & .62 \\
\hline Prior atrial septectomy & + & .08 & & & .99 \\
\hline Prior pulmonary artery banding & + & .06 & & & .29 \\
\hline Prior coarctation repair & + & .04 & & & .12 \\
\hline Preop SVT & + & .09 & & & .23 \\
\hline Preop bradyarrhythmia & + & $<.001$ & 15.8 & $3.4-72.8$ & $<.001$ \\
\hline Preop pacemaker & + & .007 & & & .84 \\
\hline Early postop SVT & + & $<.0001$ & 24.7 & $6.8-89.8$ & $<.0001$ \\
\hline Early postop bradyarrhythmia & + & .04 & & & .64 \\
\hline
\end{tabular}

$S V T$, Supraventricular tachyarrhythmia; $C I$, confidence interval; $A V V$, atrioventricular valve; $C A V V$, common atrioventricular valve.

*Risk ratio represents the odds ratio derived from logistic regression analysis for the outcome "early SVT" and hazards ratio from the Cox proportional-hazards model for the outcomes "late SVT." Only variables with a univariate $P<.1$ are shown. All variables tested are listed in the appendix.

Table IV. Predictors of bradyarrhythmia after the lateral tunnel Fontan operation

\begin{tabular}{|c|c|c|c|c|c|}
\hline \multirow[b]{2}{*}{ Variable } & \multicolumn{3}{|c|}{ Univariable } & \multicolumn{2}{|c|}{ Multivariable } \\
\hline & Association & $\mathrm{P}$ value & Risk ratio* & $95 \% C I$ & $\mathrm{P}$ value \\
\hline \multicolumn{6}{|c|}{ Early bradyarrhythmia } \\
\hline Anomalous systemic venous return & + & .05 & 3.2 & $1.3-7.9$ & .01 \\
\hline Prior pulmonary artery banding & + & .03 & & & .2 \\
\hline Preop bradyarrhythmia & + & $<.0001$ & 12.6 & $4.5-32.6$ & $<.0001$ \\
\hline Preop normofrequent junctional rhythm & + & .06 & & & .26 \\
\hline \multicolumn{6}{|c|}{ Late bradyarrhythmia } \\
\hline Prior systemic/pulmonary shunt & - & .03 & & & 6 \\
\hline Prior pulmonary artery banding & + & .001 & 3.2 & $1.3-8.4$ & .005 \\
\hline $\begin{array}{l}\text { Prior pulmonary artery-aortic anastomosis } \\
\text { or VSD enlargement }\end{array}$ & + & .05 & 2.9 & $1.2-7.5$ & .04 \\
\hline Prior bidirectional Glenn shunt & + & .05 & & & .66 \\
\hline Preop bradyarrhythmia & + & $<.0001$ & 5.1 & $2.0-13.1$ & $<.0001$ \\
\hline Preop pacemaker & + & .002 & & & .48 \\
\hline Preop Qp/Qs & + & .07 & & & .3 \\
\hline Baffle fenestration & - & .06 & & & .25 \\
\hline Early postop SVT & + & .06 & & & .29 \\
\hline Early postop bradyarrhythmia & + & $<.0001$ & 9.0 & $3.7-22.1$ & $<.0001$ \\
\hline Early postop pacing & + & .004 & & & .24 \\
\hline
\end{tabular}

$C I$, Confidence interval; VSD, ventricular septal defect; $Q p / Q s$, pulmonary/systemic flow ratio; $S V T$, supraventricular tachyarrhythmia.

*Risk ratio represents the odds ratio derived from logistic regression analysis for the outcome "early bradyarrhythmia," and hazards ratio from the Cox proportional-hazards model for the outcomes "late bradyarrhythmia." Only variables with a univariate $P<.1$ are shown. All variables tested are listed in the appendix.

lation time was 11.2 hours (range 4.7 hours to 32 days), median intensive care unit stay 2.7 days (1-93 days), and median hospital stay 10 days (4-128 days). The majority of the patients received inotropic drugs during the first 2 postoperative days. Mean arterial oxygen sat- uration at discharge was $93.7 \% \pm 3.1 \%$ (range $89 \%$ $98 \%$ ) for patients with a closed or no fenestration and $87.1 \% \pm 4.2 \%$ (range 79\%-93\%) for patients with an open fenestration. Significant pleural effusions were drained for longer than 14 days in 33 patients. 


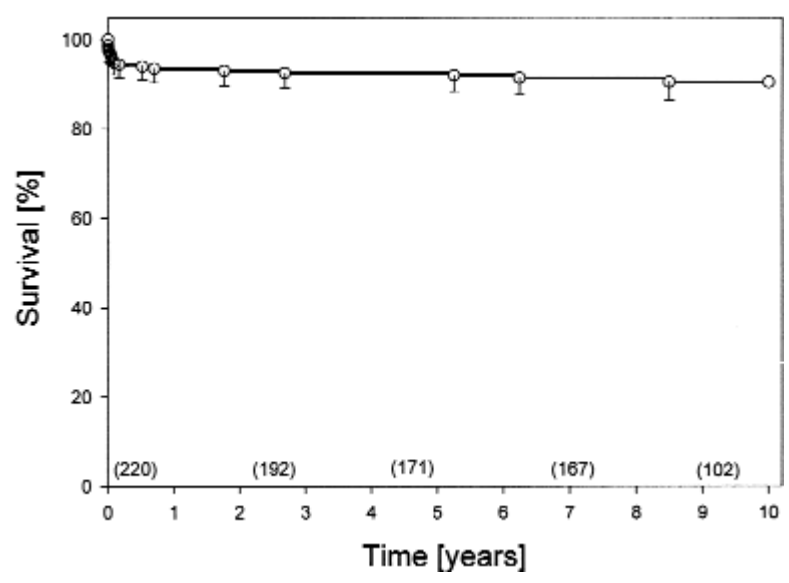

Fig 1. Kaplan-Meier estimated overall survival after the lateral tunnel Fontan operation. Error bars indicate the lower $95 \%$ confidence interval. Numbers of patients at risk are shown in parentheses.

Early postoperative ECG recordings, as well as ECGs recorded before discharge, were available for all patients. A total of 35 patients had early postoperative arrhythmias: $11(5 \%)$ patients had SVT, and 5 of those were discharged on a regimen of antiarrhythmic medication. Twenty-four (11\%) patients had bradyarrhythmia; 8 of them had a previously implanted pacemaker and 3 more required implantation of a new pacemaker before discharge. In a total of $173(83 \%)$ patients, normal sinus rhythm was documented before discharge. Independent risk factors for early SVT included heterotaxy syndrome and preoperative SVT (Table III). Anomalous systemic venous return and preoperative bradyarrhythmia were found to be predictive of early postoperative bradyarrhythmia (Table IV).

Late results. Current follow-up information regarding late mortality, morbidity, reoperations, and functional status was available in 196 of the 208 (94\%) early survivors, with a mean follow-up of $10.2 \pm 0.6$ years (median 9.5 years, range 3.1-12.3 years).

A total of 13 late failures occurred in a total of 1931 patient-years of follow-up (0.67 per 100 patient-years). There were 7 late deaths (Table I). In 1 patient the cause of death could not be elucidated. Three patients died of primary ventricular dysfunction and 1 patient of ventricular arrhythmia. One patient died of the sequelae of massive cerebral thromboembolism, and in another patient the cause of death was found to be thrombotic occlusion of the SVC and both pulmonary arteries. The Kaplan-Meier estimates of survival for the early survivors were $98 \%(97 \%-99 \%)$ at 5 years and $96 \%$ (92\%-99\%) at 10 years (Fig 1). Including early deaths, overall survival was estimated as $93 \%(89 \%-96 \%)$ at 5 years and $91 \%(86 \%-95 \%)$ at 10 years (Fig 1). In 2

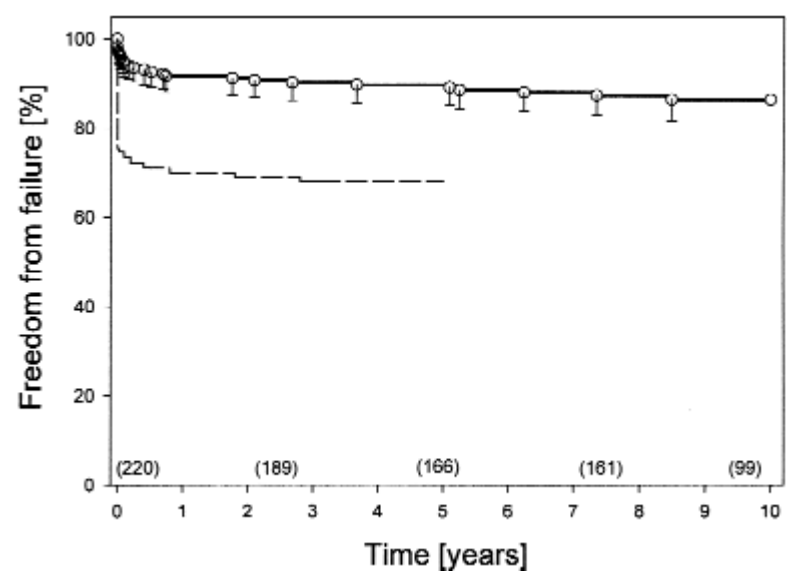

Fig 2. Kaplan-Meier estimated overall freedom from failure after the lateral tunnel Fontan operation. "Failure" includes death, takedown, and heart transplantation. Error bars indicate the lower $95 \%$ confidence interval. Numbers of patients at risk are shown in parentheses. The dashed line represents the Kaplan-Meier estimate of freedom from Fontan failure after Fontan operations performed between 1973 and 1984 in our institution, before the introduction of the lateral tunnel modification (data previously published by Gentles and associates ${ }^{10}$ ).

patients the Fontan circulation was taken down during the follow-up period for persistent pleural and pericardial effusions, in both patients by conversion to a bidirectional Glenn shunt. Four patients underwent heart transplantation, the indications being progressive ventricular dysfunction in 3 patients and thrombosis of the Fontan pathway in 1 patient with heterotaxy syndrome and complex venous anatomy (Table I). The KaplanMeier estimate of freedom from failure (defined as death or takedown) excluding early failures is $96 \%$ $(93 \%-99 \%)$ at 5 years and $92 \%(88 \%-96 \%)$ at 10 years (Fig 2). Overall freedom from failure including early failures is $90 \%(86 \%-94 \%)$ at 5 years and $87 \%(82 \%$ $91 \%$ ) at 10 years (Fig 2).

While univariable analysis identified several factors that were associated with late failure, including preoperative atrioventricular valve regurgitation, higher early postoperative right atrial pressure, and early postoperative bradyarrhythmia, multivariable analysis by means of the Cox proportional-hazards model revealed that prior coarctation repair was the only independent risk factor. Patients in whom the aorta arises from an outlet chamber or a muscular conus may also have a subtle degree of systemic ventricular outflow tract obstruction, potentially contributing to late myocardial dysfunction and Fontan failure. Six of the patients with Fontan failure had relevant subaortic stenosis, and all of them underwent a pulmonary artery-aortic anastomosis before the Fontan operation. Of the other 21 
Volume 121, Number 1

patients with Fontan failure, 11 had transposition or malposition of the great arteries and hence the potential for development of some degree of systemic ventricular outflow tract obstruction. This may indeed indicate the possibility of a subtle systemic ventricular outflow tract obstruction as a factor contributing to Fontan failure, but when a potentially predisposing anatomy was examined as a variable in the entire cohort, we did not find a significant impact on outcome. When the total number of Fontan failures was evaluated as the outcome parameter, higher postoperative right atrial pressure was found to be predictive of poorer outcome (Table II). With respect to late death only, prior pacemaker insertion was the sole multivariable predictor (hazard ratio $=16.1,95 \% \mathrm{CI}=6.0-42.8, P<.001)$. Both prior coarctation repair (hazard ratio $=8.9,95 \%$ $\mathrm{CI}=2.7-29.6, P=.003$ ) and single right ventricle (hazard ratio $=7.7,95 \% \mathrm{CI}=2.2-27.2, P=.009)$ were independently predictive of overall higher risk for death.

The baffle fenestration was closed during the followup period in 77 patients by means of a transcatheter device. The fenestration is known to have closed spontaneously in 25 patients. Two patients had a mild obstruction of the Fontan connection located at the anastomosis between the proximal SVC and the pulmonary artery; these obstructions were successfully relieved by balloon dilation and stent placement. In 11 patients a leak in the intra-atrial baffle was identified and closed by transcatheter $(n=5)$ or surgical $(n=1)$ means. Other late reoperations or reinterventions included embolization of systemic-pulmonary arterial collaterals $(n=2)$, pulmonary artery dilation $(n=5)$ or surgical plasty $(n=1)$, relief of subaortic outflow tract obstruction $(n=3)$, creation of a pericardial window (n $=4)$, and creation of a baffle fenestration $(n=1)$.

Follow-up cardiac catheterization data were available for 101 patients and echocardiograms for 130 patients. Mean aortic oxygen saturation at follow-up was $91 \% \pm$ $4.6 \%$ (77\%-97\%), and mean central venous pressure was $11.4 \pm 3.2 \mathrm{~mm} \mathrm{Hg}(7-22 \mathrm{~mm} \mathrm{Hg})$.

ECGs recorded during late follow-up were available for review in 114 (59\%) patients. In 67 (35\%) patients the information about current cardiac rhythm was obtained from filed correspondence. Holter recordings were available for 38 (20\%) patients. The median interval between the Fontan operation and most recent ECG recording was 8.7 years (range 3-12.3 years). For 12 patients no recent ECG recording or report was available. SVT was reported in $12(5.5 \%)$ patients during the follow-up period, 1 of whom was known to have a history of preoperative SVT. The overall freedom from symptomatic or documented postoperative SVT incidents was $94 \%(91 \%-97 \%)$ at 5 years and $90 \%(85 \%$ -

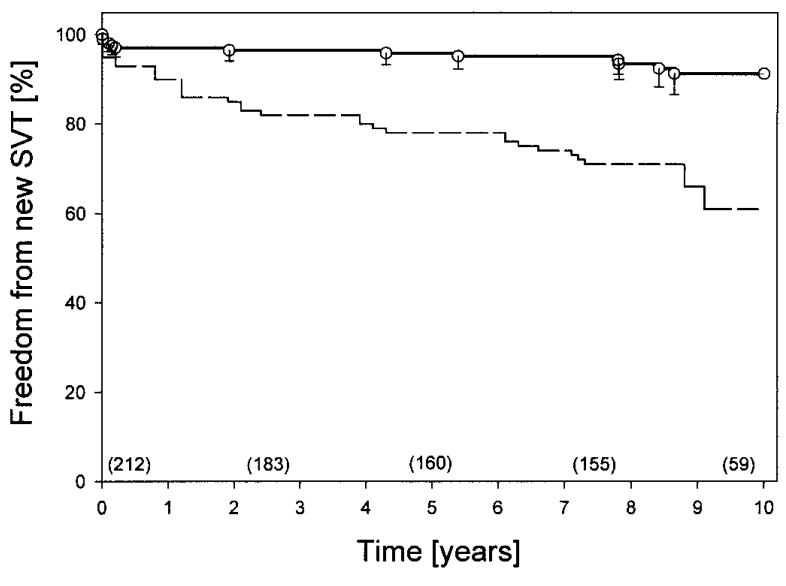

Fig 3. Kaplan-Meier estimated overall freedom from new SVT after the lateral tunnel Fontan operation. New SVT is defined as postoperative occurrence of SVT excluding patients who had preoperative SVT. Error bars indicate the lower $95 \%$ confidence interval. Numbers of patients at risk are shown in parentheses. The dashed line represents the Kaplan-Meier estimate of freedom from atrial flutter after a Fontan operation with direct atriopulmonary anastomosis in patients operated on between 1973 and 1989 in our institution (data previously published by Fishberger and associates ${ }^{12}$ ).

$95 \%)$ at 10 years. When patients with preoperative SVT are excluded, the freedom from new postoperative SVT is $96 \%(93 \%-99 \%)$ at 5 years and $91 \%(86 \%$ $96 \%$ ) at 10 years (Fig 3). Freedom from late-onset SVT is $98 \%(97 \%-99 \%)$ at 5 years and $94 \%(92 \%-97 \%)$ at 10 years. The proportions of patients having preoperative SVT (7/220, 3.2\%) and those having SVT during recent follow-up $(7 / 192,3.6 \%)$ were not different $(P=.9$, Fisher exact test). Numerous variables were associated with late SVT in the univariable analyses, including heterotaxy syndrome, anomalous systemic venous return, prior atrial septectomy, preoperative SVT, and early postoperative bradyarrhythmia. However, the Cox model established 4 important predictors: atrioventricular valve regurgitation, common atrioventricular valve, preoperative bradyarrhythmia, and early postoperative SVT (Table III). When the onset of new postoperative SVT was considered as the outcome parameter, the presence of a common atrioventricular valve was the only multivariable risk factor (hazard ratio $=4.7,95 \% \mathrm{CI}=1.5-15.6, P=.03$ ).

A total of 32 patients (15.3\%) had an incident of postoperative bradyarrhythmia documented during the follow-up period; 9 of those also had preoperative bradyarrhythmia and 11 early postoperative bradyarrhythmia. In addition to manifest bradyarrhythmias, $12(6 \%)$ patients had atrial junctional rhythm with nor- 


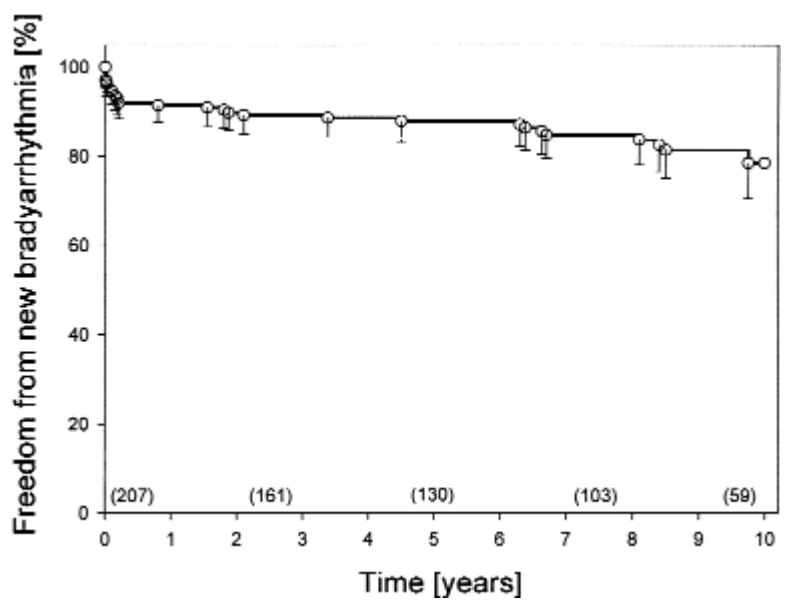

Fig 4. Kaplan-Meier estimated freedom from new bradyarrhythmia after the lateral tunnel Fontan operation. New bradyarrhythmia is defined as postoperative occurrence of bradyarrhythmia excluding patients who had preoperative bradyarrhythmia. Error bars indicate the lower 95\% confidence interval. Numbers of patients at risk are shown in parentheses.

mal heart rate at most recent follow-up; 11 of them also had preoperative junctional rhythm. Estimated freedom from new postoperative bradyarrhythmia was $88 \%$ $(83 \%-92 \%)$ at 5 years and $79 \%(72 \%-86 \%)$ at 10 years (Fig 4). Freedom from late-onset bradyarrhythmia was $94 \%(91 \%-97 \%)$ at 5 years and $89 \%(83 \%-94 \%)$ at 10 years. When patients with permanent preoperative bradyarrhythmia are included, the overall freedom from bradyarrhythmia was estimated to be $83 \%$ (83\%$92 \%)$ at 5 years and $73 \%(67 \%-79 \%)$ at 10 years. Twenty-seven (14\%) of the 192 patients had bradyarrhythmia at the time of most recent follow-up, as compared with 27 (12\%) of 220 patients who had preoperative bradyarrhythmia $(P=.42)$. Again, there were numerous univariable factors associated with late bradyarrhythmia, such as a prior bidirectional Glenn shunt and early postoperative SVT. Besides a prior pulmonary artery-aortic anastomosis or VSD enlargement and prior pulmonary artery banding, the most important independent multivariable risk factors for late bradyarrhythmia were preoperative and early postoperative bradyarrhythmia (Table IV). When the onset of new postoperative bradyarrhythmia was considered as the outcome parameter, no variables were found to be independently predictive in the Cox regression model. Enlargement of the proximal cavopulmonary anastomosis by incising the cavoatrial junction and the roof of the right-sided atrium did not prove a risk factor for early or late bradyarrhythmia.
A pacemaker was implanted during the follow-up period in 7 patients, with epicardial leads placed on the ventricular and atrial surfaces after median sternotomy. Four of those had isolated bradyarrhythmia and 3 patients had sick sinus syndrome with recurrent atrial flutter. The indications for pacemaker insertion were documented bradyarrhythmia with heart rates less than 50 beats/min in 5 patients and symptomatic bradyarrhythmic episodes with heart rates more than 50 beats/min in 2 patients.

Functional state. A New York Heart Association functional class was assigned by the primary physician or cardiologist in 183 patients. Seventy-five (41\%) of the patients were in functional class I, 97 patients $(53 \%)$ were in class II, 11 patients $(6 \%)$ were in class III, and no patient was in class IV. A medication history was available in 169 patients. One hundred twelve patients were receiving no cardiac medications at the time of follow-up, 39 patients were receiving one cardiac medication, and 18 were receiving two or more cardiac medications. Antiarrhythmic medication other than digoxin was required by 13 patients. There was no uniform policy regarding the indication for long-term anticoagulation. Twenty-seven patients with reduced ventricular function and/or chronic atrial flutter or fibrillation received warfarin sodium (Coumadin). Platelet inhibition with aspirin $80 \mathrm{mg} /$ day by mouth was performed in 118 patients.

Neurologic symptoms were present in a total of 7 patients. In 2 patients a hemiplegia developed 3 and 14 months, respectively, after the Fontan operation. Both had open fenestrations, but no source of emboli was identified. Two patients have seizures, 1 of unknown cause and 1 after a septic brain abscess. Three other patients have mild choreoathetosis symptoms, all after uncomplicated hospital courses. Apart from 2 patients with thrombotic occlusion of the SVC, 1 patient with late baffle thrombosis, 1 patient with massive cerebral thromboembolism, and 2 patients with stroke and hemiplegia, there were no proven or suspected thromboembolic complications in this series. Protein-losing enteropathy with persistent or recurrent edema, hypoproteinemia, and enteric $\alpha_{1}$-antitrypsin loss developed in 1 patient with transposition of the great arteries and Ebstein anomaly. He had moderate tricuspid valve regurgitation and underwent a modified Stansel anastomosis for subaortic obstruction 22 months after the Fontan operation. However, his protein-losing enteropathy did not improve, and he eventually underwent cardiac transplantation, resulting in complete remission. Two further patients had evidence of protein-losing enteropathy in the form of recurrent ascites and hypoproteinemia, for which they were successful- 
ly treated with diuretics and angiotensin-converting enzyme inhibitors.

\section{Discussion}

The present study demonstrates that the lateral tunnel Fontan procedure results in excellent long-term survival and freedom from failure in patients with a wide range of underlying cardiac malformations. The incidence of new SVT is low, but a number of patients will have some form of bradyarrhythmia during follow-up. However, a large proportion of lateral tunnel Fontan patients with bradyarrhythmia already had sinus node dysfunction before the operation, mainly associated with structural anomalies or previous surgical procedures.

Although the surgical results of the Fontan operation for complex cardiac malformations are steadily improving, it has become evident that considerable late morbidity develops with increasing follow-up time. ${ }^{10,16-18}$ This has been demonstrated clearly for the atriopulmonary types of Fontan circulation but remains to be defined for more recent modifications, such as the lateral tunnel and the total extracardiac Fontan operation. There is experimental evidence that the intra-atrial suture line alone is able to facilitate inducible sustained atrial flutter ${ }^{19}$ and that the fluid dynamics in the lateral tunnel are characterized by slightly greater fluid-power loss compared with the extracardiac cavopulmonary connection..$^{20}$ We therefore chose to determine the longterm results in a group of patients invariably treated with a lateral tunnel Fontan operation, which, in our institution, is used most often.

Although late mortality and morbidity in our series are low, a significant number of patients have late postoperative bradyarrhythmia. Because of the mostly permanent nature of bradyarrhythmias, preoperative and early postoperative bradyarrhythmia proved to be the most potent predictors of late bradyarrhythmia, followed by structural anomalies of the systemic venous return, a feature of many malformations that are associated with abnormalities of the conduction system. Regarding the 2 procedure-related variables with significant impact on the prevalence of late bradyarrhythmia, prior pulmonary artery banding and prior pulmonary artery-aortic anastomosis, there seems to be no obvious cause-effect relationship. A staged approach to completion of the Fontan circulation was previously found to increase the risk of postoperative bradyarrhythmia, but in the current analysis a prior bidirectional Glenn shunt proved to be a risk factor in the univariable analysis only. ${ }^{21}$ However, it is notable that 7 of the 10 patients with preoperative junctional rhythm at a physiologic rate had had a previous Glenn shunt. As pointed out before, the proximal cavopulmonary anastomosis was enlarged by incising the roof of the rightsided atrium in patients with a relatively small SVC orifice. An incision across the superior cavoatrial junction may damage the sinus node artery, since the vessel is difficult to identify during cardioplegic arrest. Interestingly, however, we did not observe a correlation between an incision across the cavoatrial junction and the incidence of postoperative bradyarrhythmia.

Exposure of the entire atrium to elevated pressure in the Fontan circuit can lead to progressive distention with atrial enlargement and development of supraventricular arrhythmias. Whether incorporation of only a small area of the lateral atrial wall in the Fontan circulation, as in the lateral tunnel modification, has similar deleterious effects on atrial conduction remains unclear. The presence of long intra-atrial suture lines as necessary for implantation of the lateral tunnel is thought to facilitate the development of slow reentry circuits and hence atrial flutter. ${ }^{19}$ This has indeed been demonstrated by intra-atrial mapping and programmed stimulation in a number of patients after the lateral tunnel Fontan operation. The proportion of patients in whom reentry circuits actually lead to arrhythmias is not completely clear. However, our data indicate that very few patients have symptomatic tachycardias within the first 10 years after the operation. When Fishberger and associates ${ }^{12}$ presented the Children's Hospital Boston experience with arrhythmias after the modified Fontan operation, they noted a trend to a lower incidence of postoperative tachyarrhythmias after a lateral tunnel Fontan operation as compared with other modifications. Because of the limited number of patients and short follow-up, the difference did not reach statistical significance. However, in the present study we could clearly demonstrate that the low incidence of SVT persists for at least up to 10 years after the lateral tunnel Fontan procedure. Reviewing the extensive literature on the development of supraventricular arrhythmia after the Fontan operation, we noticed that the incidence of SVT after lateral tunnel Fontan modifications in other studies is also low. ${ }^{3,6,22}$ This suggests that, although the suture line may be highly arrhythmogenic in animal models, other factors may play a more important role in the clinical evolution of this arrhythmia. Only one report, a comparative study from the Mayo Clinic, suggests that patients after a lateral tunnel Fontan are at the same risk for early and late SVT as patients with other types of Fontan connections. ${ }^{23}$ Moreover, the risk for late SVT after a total cavopulmonary connection was higher than after an atriopulmonary connection with an intra-atrial lateral tunnel. 
The proponents of a total extracardiac Fontan procedure, using a tubular conduit or an extracardiac tunnel incorporating atrial wall, have suggested that this modification may minimize the incidence of atrial arrhythmia. ${ }^{24-26}$ Although several theoretical arguments support this hypothesis, the detailed study by Shirai and colleagues $^{27}$ demonstrated that 11 of 15 patients after an extracardiac Fontan operation had some form of bradyarrhythmia at follow-up. In addition, 3 patients had a thrombus located in the external Fontan conduit. In our series, heterotaxy syndrome proved the only patient-related risk factor for early SVT in the multivariable model and a univariable risk factor for late SVT. Patients with heterotaxy syndrome have consistently been shown to be at greater risk of both early and late mortality and morbidity. ${ }^{28,29}$ Numerous abnormalities of the conduction system exist that may provoke tachyarrhythmia and bradyarrhythmia irrespective of the type of Fontan repair. In patients with left isomerism, interrupted IVC, and azygos continuation, we prefer to use a lateral tunnel to direct the hepatic venous flow to the cavopulmonary anastomosis whenever the intra-atrial anatomy allows. This is important to avoid the development of pulmonary arteriovenous communications due to a lack of "hepatic factor" in the pulmonary circulation. Since blood flow through the conduit from hepatic veins only is lower than from a regular IVC, a tubular intracardiac or extracardiac conduit may be at increased risk of thrombosis.

The risk factors for failure of the Fontan circulation and the proposed selection criteria for a Fontan-type repair have been described frequently. However, many of the exclusion criteria have been relaxed with improving perioperative management and surgical modifications. The present cohort was operated on within a relatively narrow time window with one principal surgical technique with only slight modifications. Hence, it is not surprising that early outcome was only determined by "classic" patient-related risk factors such as a single right ventricle, pulmonary artery distortion, pulmonary vascular resistance, and postoperative right atrial pressure. Although our principal treatment strategy has since been unchanged, it is likely that further improvements in perioperative management during the past decade, such as better defined indications for insertion and closure of the baffle fenestration or the use of inhaled nitric oxide during the early postoperative period, have further limited the number of early risk factors. ${ }^{30,31}$ The long-term outcome is more likely to reflect advances in surgical technique, and the only independent predictor of late failure was prior coarctation repair. Whether this surprising finding is incidental or reflects late effects of early systemic ventricular pressure overload remains to be determined. Another explanation would be residual or recurrent coarctation in these patients. However, we did not find any evidence for that in patients with a failing Fontan circulation.

\section{Limitations of the study}

The validity of our study is clearly limited by its retrospective nature. Although long-term follow-up information was available for a large number of patients, the incidence of bradyarrhythmia and transient SVT is most likely underestimated because of the transient and intermittent nature of some arrhythmias and the limited availability of serial ECG and Holter studies. On the other hand, we did not distinguish between symptomatic and asymptomatic arrhythmias, and only a fraction of the recorded arrhythmic events required treatment. Similarly, the incidence of thromboembolic complications is probably underestimated, because only clinically relevant events came to our attention. For instance, Balling and associates ${ }^{32}$ have recently reported right atrial thrombus formation in $33 \%$ of asymptomatic patients having the Fontan operation, although all of the thrombus-positive patients had either atriopulmonary or atrioventricular Fontan modifications. The same must be said regarding the incidence of protein-losing enteropathy, which can be better assessed by prospective analysis of enteric $\alpha_{1}$-antitrypsin loss. Since the absolute number of late events is small, further caution is recommended when interpreting the results of the risk factor analyses. With respect to the early results, it should also be remembered that the cohort represents our early experience with the lateral tunnel Fontan operation, and, as pointed out before, further advances in early postoperative treatment have probably led to a further decrease in early mortality and morbidity. Our retrospective analysis can by no means replace prospective studies of long-term Fontan complications such as arrhythmias, thromboembolism, or organ dysfunction. Instead, it may serve as a basis for the design of future studies clarifying the long-term morbidity of patients with modern Fontan modifications.

\section{Conclusions}

By extending the limits for the Fontan procedure, we treat many patients with severe cardiac malformations that carry an intrinsic higher risk of late morbidity and mortality irrespective of the nature of palliative procedures. ${ }^{18}$ On the basis of favorable long-term outcome, the lateral tunnel Fontan operation remains our pre- 
ferred method to establish a Fontan circulation for a wide range of underlying cardiac malformations. However, further prospective studies clarifying the long-term morbidity of modern Fontan modifications are clearly needed.

\section{REFERENCES}

1. Fontan F, Baudet E. Surgical repair of tricuspid atresia. Thorax 1971;26:240-8.

2. Kreutzer G, Galindez E, Bono H, De Palma C, Laura JP. An operation for the correction of tricuspid atresia. J Thorac Cardiovasc Surg 1973;66:613-21.

3. Balaji S, Gewillig M, Bull C, de Leval MR, Deanfield JE. Arrhythmias after the Fontan procedure: comparison of total cavopulmonary connection and atriopulmonary connection. Circulation 1991;84(Suppl):III-162-7.

4. Driscoll DJ, Offord KP, Feldt RH, Schaff HV, Puga FJ, Danielson GK. Five- to fifteen-year follow-up after Fontan operation. Circulation 1992;85:469-96.

5. Knott-Craig CJ, Danielson GK, Schaff HV, Puga FJ, Weaver AL, Driscoll DD. The modified Fontan operation: an analysis of risk factors for early postoperative death or takedown in 702 consecutive patients from one institution. J Thorac Cardiovasc Surg 1995; 109:1237-43

6. Gelatt M, Hamilton RM, McCrindle BW, Gow RM, Williams WG, Trusler GA, et al. Risk factors for atrial tachyarrhythmias after the Fontan operation. J Am Coll Cardiol 1994;24:1735-41.

7. Gewillig M, Wyse RK, de Leval MR, Deanfield JE. Early and late arrhythmias after the Fontan operation: predisposing factors and clinical consequences. Br Heart J 1992;67:72-9.

8. de Leval MR, Kilner P, Gewillig M, Bull C. Total cavopulmonary connection: a logical alternative to atriopulmonary connection for complex Fontan operations-experimental studies and early clinical experience. J Thorac Cardiovasc Surg 1988;96:682-95.

9. Jonas RA, Castaneda AR. Modified Fontan procedure: atrial baffle and systemic to pulmonary artery anastomotic techniques. J Card Surg 1988;3:91-6.

10. Gentles TL, Mayer JE, Gauvreau K, Newburger JW, Lock JE, Kupferschmid JP, et al. Fontan operation in five hundred consecutive patients: factors influencing early and late outcome. J Thorac Cardiovasc Surg 1997;114:376-91.

11. Gentles TL, Gauvreau K, Mayer JE Jr, Fishberger SB, Burnett J, Colan SD, et al. Functional outcome after the Fontan operation: factors influencing late morbidity. J Thorac Cardiovasc Surg 1997;114:392-403.

12. Fishberger SB, Wernovsky G, Gentles TL, Gauvreau K, Burnett J, Mayer JE Jr, et al. Factors that influence the development of atrial flutter after the Fontan operation. J Thorac Cardiovasc Surg 1997;113:80-6

13. Kreutzer J, Keane JF, Lock JE, Walsh EP, Jonas RA, Castaneda $\mathrm{AR}$, et al. Conversion of modified Fontan procedure to lateral atrial tunnel cavopulmonary anastomosis. J Thorac Cardiovasc Surg 1996;111:1169-76.

14. Kugler JD. Sinus node dysfunction. In: Gillette PC, Garson A Jr, editors. Pediatric arrhythmias: electrophysiology and pacing. Philadelphia: WB Saunders; 1990. p. 250-300.

15. Hosmer DW, Lemeshow S. Applied survival analysis: regression modeling of time to event data. New York: John Wiley; 1999. p. 40-55.
16. Cetta F, Feldt RH, O'Leary PW, Mair DD, Warnes CA, Driscoll DJ, et al. Improved early morbidity and mortality after the Fontan operation: the Mayo Clinic experience, 1987 to 1992. J Am Coll Cardiol 1996;28:480-6.

17. Mayer JE Jr, Bridges ND, Lock JE, Hanley FL, Jonas RA, Castaneda AR. Factors associated with marked reduction in mortality for Fontan operations in patients with single ventricle. J Thorac Cardiovasc Surg 1992;103:444-51.

18. Mayer JE Jr, Helgason H, Jonas RA, Lang P, Vargas FJ, Cook N, et al. Extending the limits for modified Fontan procedures. J Thorac Cardiovasc Surg 1986;92:1021-8.

19. Rodefeld MD, Bromberg BI, Schuessler RB, Boineau JP, Cox JL, Huddleston CB. Atrial flutter after the lateral tunnel construction in the modified Fontan operation: a canine model. J Thorac Cardiovasc Surg 1996;111:514-26.

20. Lardo AC, Webber SA, Friehs I, del Nido PJ, Cape EG. Fluid dynamic comparison of intra-atrial and extracardiac total cavopulmonary connections. J Thorac Cardiovasc Surg 1999;117:697-704.

21. Manning PB, Mayer JE Jr, Wernovsky G, Fishberger SB, Walsh EP. Staged operation to Fontan increases the incidence of sinoatrial node dysfunction. J Thorac Cardiovasc Surg 1996;111:833-9.

22. Gardiner HM, Dhillon R, Bull C, de Leval MR, Deanfield JE. Prospective study of the incidence and determinants of arrhythmia after total cavopulmonary connection. Circulation 1996;94(Suppl):II-17-21.

23. Durongpisitkul K, Porter CJ, Cetta F, Offord KP, Slezak JM, Puga FJ, et al. Predictors of early- and late-onset supraventricular tachyarrhythmias after Fontan operation. Circulation 1998;98:1099-107.

24. Marcelletti C, Corno A, Giannico S, Marino B. Inferior vena cava-pulmonary artery extracardiac conduit: a new form of right heart bypass. J Thorac Cardiovasc Surg 1990;100:228-32.

25. Amodeo A, Galletti L, Marianeschi S, Picardo S, Giannico S, Di Renzi P, et al. Extracardiac Fontan operation for complex cardiac anomalies: seven years' experience. J Thorac Cardiovasc Surg 1997;114:1020-30.

26. Petrossian E, Reddy VM, McElhinney DB, Akkersdijk GP, Moore P, Parry AJ, et al. Early results of the extracardiac conduit Fontan operation. J Thorac Cardiovasc Surg 1999;117:688-96.

27. Shirai LK, Rosenthal DN, Reitz BA, Robbins RC, Dubin AM. Arrhythmias and thromboembolic complications after the extracardiac Fontan operation. J Thorac Cardiovasc Surg 1998;115: 499-505.

28. Humes RA, Feldt RH, Porter CJ, Julsrud PR, Puga FJ, Danielson GK. The modified Fontan operation for asplenia and polysplenia syndromes. J Thorac Cardiovasc Surg 1988;96:212-8.

29. Michelion G, Gharagozloo F, Julsrud PR, Danielson GK, Puga FJ. Modified Fontan operation in the presence of anomalies of systemic and pulmonary venous connection. Circulation 1993;88(Suppl):II-141-8.

30. Bridges ND, Mayer JE Jr, Lock JE, Jonas RA, Hanley FL, Keane $\mathrm{JF}$, et al. Effect of baffle fenestration on outcome of the modified Fontan procedure. Circulation 1992;86:1762-9.

31. Bridges ND, Lock JE, Mayer JE Jr, Burnett J, Castaneda AR. Cardiac catheterization and test occlusion of the interatrial communication after the fenestrated Fontan operation. J Am Coll Cardiol 1995;25:1712-7.

32. Balling G, Vogt M, Kaemmerer H, Eicken A, Meisner H, Hess J. Intracardiac thrombus formation after the Fontan operation. $\mathrm{J}$ Thorac Cardiovasc Surg 2000;119:745-52. 


\section{Appendix}

Variables tested for influence on early and late outcome parameters

Patient-related

Demographics

Age at Fontan operation

Sex

Time since Fontan operation

Diagnosis

Morphologically left ventricle with normally related great arteries

Morphologically left ventricle with transposed great arteries

Morphologically right ventricle and ventricle with unknown morphology

Hypoplastic left heart syndrome

Heterotaxy syndrome

Anomalous pulmonary venous drainage

Atrioventricular valve regurgitation

Pulmonary artery distortion

Atrioventricular valve morphology

Left atrioventricular valve stenosis or atresia

Right atrioventricular valve stenosis or atresia

Double-inlet ventricle

Common atrioventricular canal

Pulmonary artery distortion

Prior procedures

Systemic-pulmonary artery shunt

Pulmonary artery band

Coarctation repair (including hypoplastic left heart syndrome with distinct coarctation)

Atrial septectomy

Pulmonary artery-aortic anastomosis or VSD enlargement

Atrioventricular valve repair

Bidirectional Glenn shunt or hemi-Fontan operation

Pulmonary artery reconstruction

Pacemaker implantation

Preoperative hemodynamics

Arterial oxygen saturation

Hemoglobin

Left atrial pressure

Right atrial pressure

Mean pulmonary artery pressure

Pulmonary vascular resistance

Pulmonary/systemic flow ratio

Pulmonary plus systemic flow

Preoperative supraventricular tachyarrhythmia

Preoperative bradyarrhythmia

Preoperative atrial junctional rhythm

Procedure-related variables

Cardiopulmonary bypass time

Aortic crossclamp time

Surgeon

Concomitant procedures

Baffle fenestration

Incision of cavoatrial junction/atrial roof

Atrial septectomy

Pulmonary arterioplasty

Atrioventricular valve repair

Relief of subaortic obstruction

Postoperative variables

Right atrial pressure on postoperative day 1

Duration of intensive care unit stay

Early postoperative SVT

Early postoperative bradyarrhythmia
Early postoperative atrial junctional rhythm

Early postoperative ventricular pacing

Early postoperative atrial pacing

Early postoperative atrioventricular pacing

Fenestration closure

Pleural effusions $>14$ days

Need for catheter or surgical intervention other than fenestration

closure

Duration of hospital stay

\section{Discussion}

Dr Gordon K. Danielson (Rochester, Minn). Dr Stamm has reminded me that I was privileged to discuss Marc de Leval's now classic presentation on the hydrodynamics of total pulmonary connection at the 1988 meeting of this Association. Some of the issues raised at that time have been answered by Dr Stamm's detailed review and by reports of others, specifically the reduced incidence of right atrial thrombus in the more streamlined atrial channel and a lower incidence of atrial tachyarrhythmias due to exclusion of most of the right atrium from increased systemic venous pressure. Dr Stamm's review confirms the views that there are also other factors causally associated with atrial tachyarrhythmias, including some on the systemic side of the circulation, particularly atrioventricular valve abnormalities and incompetence.

It is difficult to prove that the presence and location of atrial suture lines are important risk factors for atrial tachyarrhythmias in Fontan patients, although increasing evidence suggests that they may be. For example, there are several ways to construct a lateral tunnel. In our experience, patients with a baffle sewn to the crista terminalis had nearly twice the incidence of late atrial tachyarrhythmias as did those with a baffle sewn anterior to and well removed from the crista terminalis. The authors point out that Dr James Cox and his colleagues have shown experimentally that injury to the crista terminalis produces a substrate for atrial flutter.

My first question for Dr Stamm is as follows: Was the location of the atrial baffle suture line in reference to the crista mentioned in the operative notes? If so, was it possible to compare cases with and without suture lines in the crista?

With regard to bradyarrhythmias, there was a 10-year probability of the development of a new bradyarrhythmia of $21 \%$ in your series. In 62 patients an incision was made down the SVC and across the roof of the right atrium, probably injuring the sinus node blood supply. This variable was not listed in your Table IV, but in the discussion you mentioned that a correlation was not noted between this incision and the incidence of postoperative bradyarrhythmias. Can you explain this counterintuitive finding?

Incidentally, one advantage of the lateral tunnel compared with an extracardiac or intracardiac tube is the ability to place a transvenous atrial pacemaker for this progressively enlarging subset of patients with atrial arrhythmias. Some issues regarding the lateral tunnel total cavopulmonary connection that are still undecided, at least in my understanding, include the long-term effect of offsetting the caval anastomoses in regard to pulmonary arteriovenous fistulas in the lung that receives little or no hepatic flow, as well as the importance 
and tradeoffs regarding the best way to place and fashion the cavopulmonary anastomoses. This excellent analysis of the early and late results of the intracardiac lateral tunnel Fontan procedure will serve well as a basis for comparisons with the other surgical modifications.

Dr Stamm. Thank you very much for your comments, Dr Danielson.

With respect to your first question, I would like to refer to Dr Mayer's excellent presentation in the postgraduate course that immediately preceded this meeting, in which he explained in detail the technique that is currently used at Children's Hospital. My understanding is that we prefer not to suture the intra-atrial baffle to the crista terminalis itself, being aware of the possible increased incidence of arrhythmia that might result.

With respect to your second question, we did indeed study whether the incision in the roof of the right atrium had an impact on the incidence of sinus node dysfunction, which it did not have. We will add that information to the manuscript.

\section{Notice of correction}

In the October 2000 issue of the Journal, in the article by Alexiou and associates titled "Double-Barreled Conduit for Right Atrioventricular Connection in Tricuspid Atresia: A New Technique" (2000;120:820-2), an error was made. On page 821 the company name "Shelhigh" was misspelled "Shelheigh" (first paragraph, first and third lines). 